\title{
Gesundheit in der Schweiz-Nationaler Gesundheitsbericht 2008
}

\author{
Verlag Hans Huber, Bern 2008, 374 S., Kt., \\ http://www.verlag-hanshuber.com/vkat/einzeltitel.php?isbn=978-3-456-84626-2, \\ ISBN: 978-3-456-84626-2
}

\author{
Katharina Meyer
}

Published online: 4 March 2009

(C) Springer-Verlag 2009

\section{Health in Switzerland}

\section{- $\quad$ The 2008 National Health Report}

In health-care discussions of recent years, it has often been assumed that many of the new chronic diseases are in the first instance triggered by individual behaviour. This led to the idea that whoever eats poorly or is physically inactive will become ill and finally to the conclusion that people only have themselves to blame. Research is now showing more and more conclusively that an individual's health is also determined by external influences. These include socio-economic factors, such as education, profession and income, as well as socio-cultural determinants, such as gender, ethnic origin or social status. How healthy a society is depends therefore on individual as well as social conditions.

Identifying relationships and multi-sector solutions

The Report "Health in Switzerland" describes for the first time the health of the population in light of these social health determinants. Switzerland, following Sweden and Finland, is one of the first countries in Europe to take up this new concept. With this move comes the conviction that a health policy has to provide more than a mere guarantee to care for sick people. A health policy should also contribute to shaping social conditions so that as many people as possible have access to health system resources. In so doing, it will also be valuable in reducing health disparities.

K. Meyer $(\bowtie)$

Swiss Health Observatory \& Medical University of Bern,

2010 Neuchâtel, Switzerland

e-mail: meyer.katharina@bluewin.ch
Such an attempt requires a health report like this one not only simply to collect data in order to show the health condition of a society, but also to show how health and illness are distributed within the socio-economic groups, with men and women considered separately and viewed over a lifespan from childhood to old age. The Report "Health in Switzerland" attempts to do this by taking into account the influence of determinants such as status, education, social resources as well as the family and social environment. It refers to the relationships that should make it possible for all persons in the public health service to develop future improvements for the health system. Such an approach shows that the objective of healthy life concerns not only health policy in the narrowest sense, but also that this is a multi-sector task involving the accountability of many societal decision-makers.

\section{Contents of the health report}

The "Health in Switzerland-National Health Report 2008" describes in five main chapters the topics that Swiss Health and Society Policy will be concerned with in the future.

- Chapter I introduces the concept of health determinants.

- Chapter II handles the health of the population at different stages of life and under difficult living conditions.

- Chapter III provides a summary of chronic illnesses and accidents.

- Chapter IV discusses the basic health determinants "education" and "environment". 
- Chapter V provides information on the utilisation, cost and financing of the health-care system.

- The conclusion describes ways to achieve a multi-sector health policy.

Each of these main chapters is divided into several sub-chapters according to subject area. A summary of the main points is provided for each sub-chapter. In conclusion, the report presents a scientific summary underlining once more the importance of the strategy "health in all areas of society and politics". The national health report therefore wishes to create a bridge between science and politics and to contribute to current discussions on health policy. 